\title{
Studies of high order modes in asymmetric dual-axis cavity
}

Cite as: Appl. Phys. Lett. 113, 243503 (2018); https://doi.org/10.1063/1.5060725

Submitted: 21 September 2018 . Accepted: 24 November 2018 . Published Online: 11 December 2018

M. Topp-Mugglestone, I. V. Konoplev (D), H. Zhang, and A. Seryi
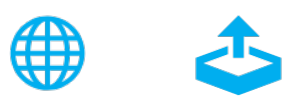

Export Citation

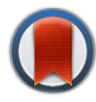

View Online

\section{ARTICLES YOU MAY BE INTERESTED IN}

Multifocal point beam forming by a single ultrasonic transducer with 3D printed holograms Applied Physics Letters 113, 243502 (2018); https://doi.org/10.1063/1.5058079

Maximizing the quality factor to mode volume ratio for ultra-small photonic crystal cavities Applied Physics Letters 113, 241101 (2018); https://doi.org/10.1063/1.5064468

Compact acoustic double negative metamaterial based on coexisting local resonances Applied Physics Letters 113, 244101 (2018); https://doi.org/10.1063/1.5052026

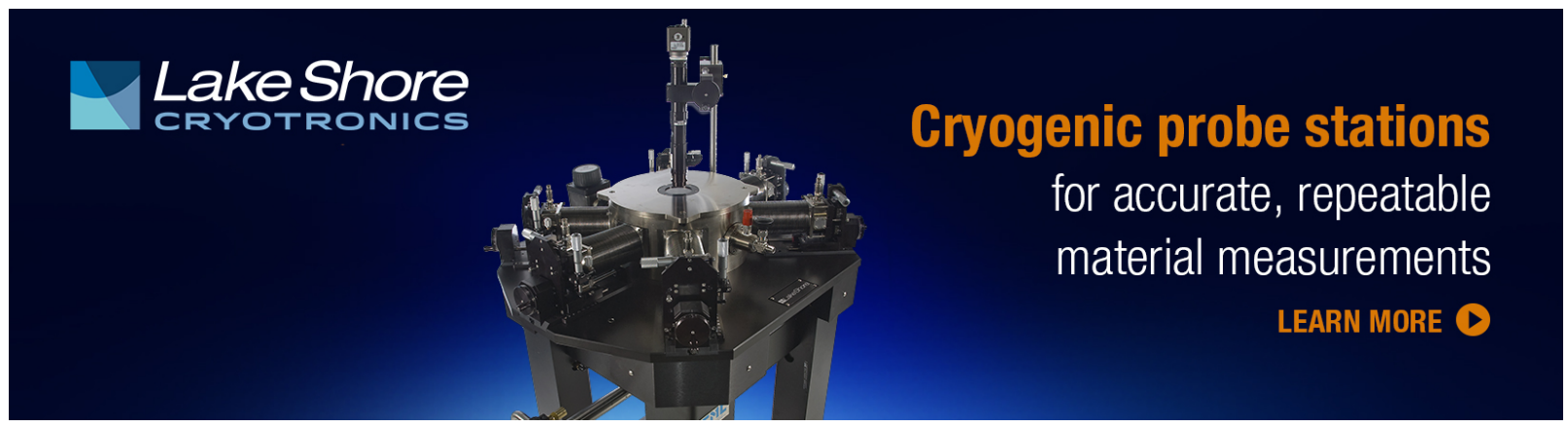




\title{
Studies of high order modes in asymmetric dual-axis cavity
}

\author{
M. Topp-Mugglestone, ${ }^{1}$ I. V. Konoplev, ${ }^{1, a)}$ H. Zhang, ${ }^{1}$ and A. Seryi ${ }^{2}$ \\ ${ }^{1}$ JAI, Department of Physics, University of Oxford, DWB, Keble Road, Oxford OXI 3RH, United Kingdom \\ ${ }^{2} J$ Lab, 12000 Jefferson Avenue, Newport News, Virginia 23606, USA
}

(Received 21 September 2018; accepted 24 November 2018; published online 11 December 2018)

\begin{abstract}
Electron-beam current in superconducting radiofrequency energy recovery linear accelerators (SCRF ERLs) is limited by beam break-up (BBU) instabilities disrupting beam transport and energy recuperation. The instabilities originate from the accumulation of high order modes (HOMs) in the cavity and positive feedback between the beam and HOMs which is especially evident in multi-pass ERLs. Overcoming the beam current limitations due to BBU instabilities is one of the challenges in accelerator science. A dual-axis asymmetric SCRF ERL has been proposed as a possible way to drive a high average current electron beam for next-generation light sources and beam cooling in electron-ion colliders (EICs). In this work, detailed studies on HOMs in the asymmetric dual axis cavity were carried out. The asymmetric field distribution of HOMs (i.e., confinement to one or another axis) has been demonstrated and their excitation from different axes has been studied. The results confirm the numerical predictions and theory developed to describe the asymmetric dual axis cavity. Published by AIP Publishing. https://doi.org/10.1063/1.5060725
\end{abstract}

Beam acceleration can be achieved using radiofrequency (RF) cavities shaped to produce the desired accelerating field profile along the axis. In an energy recovery LINAC, a beam of electrons is accelerated, used, and recirculated back into the cavity. The recirculated particles arrive $180^{\circ}$ out of phase with those that are being accelerated and are hence decelerated. Conventional energy recovery linear accelerator (ERL) systems have been designed to make use of the same RF cavities for both acceleration and energy recuperation. ${ }^{1-7}$ The use of the same cavity for both processes leads to accumulation of parasitic high order modes (HOMs), affecting the beam dynamics and limiting the beam current to around $0.5 \mathrm{~A}$ due to beam break-up (BBU) instability. To overcome the limitations imposed by the instabilities, the use of a dual axis ${ }^{8-10}$ asymmetric cavity has been suggested. ${ }^{11-14}$ The asymmetric configuration exploits the splitting of the accelerating and decelerating processes onto two slightly different sets of cavity cells centred on two parallel axes and resonantly coupled by a bridge cell [Fig. 1(a)]. The set-up allows (as shown in numerical simulations ${ }^{13}$ ) the aversion of mutual excitation of HOMs, minimising feedback between accelerating and decelerating beams and increasing BBU instability start current.

The predicted advantage of the dual-axis asymmetric cavity is the possibility of confining HOMs to either accelerating or decelerating sections implying a decaying electromagnetic field on one of the axes. This would prevent the feedback loop between sets of cells positioned on different axes becoming closed, thereby achieving the overall goal of increasing BBU start current and allowing the average current driven through such an ERL to be dramatically increased ${ }^{13,14}$ whilst meeting efficiency requirements via the use of an energy recovery stage. This is desirable for next generation high power light sources capable of generating

\footnotetext{
${ }^{\text {a) }}$ Author to whom correspondence should be addressed: ivan.konoplev@ physics.ox.ac.uk
}

high brilliance light in the $\mathrm{THz}$ and $\mathrm{X}$-ray frequency range $^{16-22}$ as well as for beam cooling in electron-ion colliders. In previous works, ${ }^{13,14}$ theoretical and preliminary experimental studies of modes in the pass band have been carried out and some confirmation of the validity of the numerical model has been demonstrated. In this paper, the measurements and rigorous experimental studies of HOMs have been conducted. Improvements of the test set-up and data analysis to allow HOM studies have been carried out and improved signal-to-noise sensitivity is demonstrated. The results observed using the improved set-up are compared with the previous measurements, and significant improvements are demonstrated.

In this paper, we show that the predictions made about the localisation of HOMs have been verified experimentally, and the results of the experimental studies are shown and discussed. We show that each HOM is localised to one of the

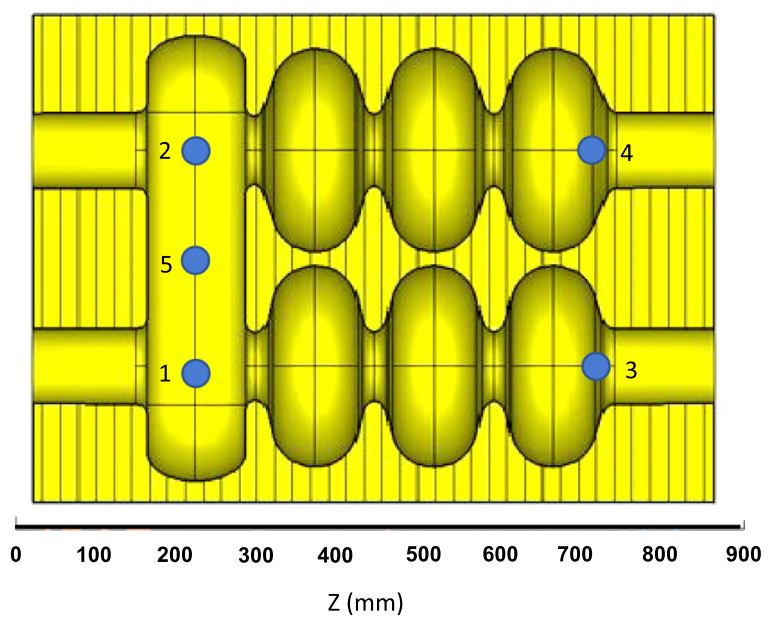

FIG. 1. Diagram of the 7-cell cavity with ports and length scale is shown. The ports' locations are schematically indicated by the blue circles and referred throughout the paper by numbers which are linked to the system axis 1-3 and 2-4 as labelled in the diagram. 
axes, with all HOMs having very different field structures inside the acceleration and deceleration sections of the cavity. The excitations of asymmetric modes including excitations on different axes have been discussed, and the results are shown. We demonstrate that HOM structures do not depend on the positioning of the couplers used to excite them along the axis, confirming numerical studies. In this work, the measurements of HOMs inside such a cavity illustrate the asymmetricity of HOMs (which is essential to enable the operation of high current ERL), improving the understanding of the HOM distributions and confirming the validity of the numerical studies. The results are the next step towards the development of the high average current ERLs needed for both fundamental research and industrial applications.

The RF bead pull test procedure is based on the introduction of a small-volume (compared with the operating wavelength or volume of the cavity) and typically dielectric object into a resonant cavity, resulting in a perturbation of the stored energy within the cavity. The perturbation to the energy stored varies as the dielectric object is moved from the high intensity field of the mode to its zero. Measuring the complex responses of the $S_{11}$ and $S_{12}$ parameters, one can observe sufficient information about the eigenmode field structure inside the cavity. The measurements of the phase shift $\varphi$ that occurs when the perturbation is introduced can be used to obtain the shift in resonant frequency $\Delta f$

$$
\frac{\Delta f}{f}=\frac{\tan \varphi}{2 Q},
$$

where $f$ is the resonance frequency of the unperturbed mode, $\varphi$ is the measured phase shift relative to that of the unperturbed mode, and $Q$ is the quality factor of the unperturbed mode. The resonant frequency shift is proportional to the square of the local E-field at the position of the object, and for a small (i.e., E field variations are small over the scale of the bead) spherical dielectric bead, the following relation between the field amplitude and the shift of the frequency is true:

$$
U \frac{\Delta f}{f}=\frac{\varepsilon_{r}-1}{\varepsilon_{r}+2} \varepsilon_{0} E_{0}^{2},
$$

with $U$ as the total energy stored in the cavity, $E_{0}$ as the local electric field for the unperturbed cavity, $\varepsilon_{r}$ as the relative permittivity of the bead, and all other symbols having their usual meaning. It is clear that if $\varepsilon_{r} \gg 2$, expression (2) can be simplified to avoid uncertainties with the value of the relative permittivity and improve the sensitivity of the measurements. In the set of experiments discussed, a probe made of PMMA (acrylic glass) with $\varepsilon_{r}=3.4$ was used. The 7-cell test cavity (Fig. 1) $)^{13,14}$ was set-up upon an optical table, which was vibrationally isolated so environmental impact on the measurements could be minimised. Additionally, the measurements were carried out overnight to avoid other interferences.

A pair of electric dipole probes (couplers) connected to the two-port VNA were inserted through specific cavity ports, and both couplers were used to excite and probe the
EM field inside the cavity. As opposed to the previous setups $^{14}$ for these experiments, the couplers were inserted vertically through ports which were vertically aligned with the cavity axes, located along the bridge cell and at the opposite side of the cavity, as shown by the circles in Fig. 1. In Fig. 1, the technical drawings of the cavity and schematic position of the five ports (blue circles) used to study the HOMs and pass-band modes are shown. We note that the location of the couplers vertically and not along the axis improved significantly the quality of the results observed and their reproducibility. To locate eigenmode frequencies, the amplitude of the transmission and reflection coefficients was measured, i.e., both $S_{11}$ and $S_{12}$ parameters. Frequency-response measurements were performed between 1 and $2.5 \mathrm{GHz}$; peaks in the frequency-response graph were taken to represent resonance modes. At each identified mode, the amplitude of the E-field (2) along each axis of the system is measured using the bead pull method ${ }^{15}$ with a step of $5 \mathrm{~mm}$ whilst the argument of the $S_{21}$ parameter is measured repeatedly (to mitigate the impact of small timescale random noise due to vibrations). The tangent of the phase shift gives the change in the frequency corresponding to the perturbation introduced by the dielectric bead, which is proportional to the local $E_{0}$ field at the location of the bead [see expressions (1) and (2)]. The mean phase shift is used to deduce the local $E_{O}$ field along the z-axis, and we normalise the field detected outside the cavity to 1 to obtain the relative magnitude of the E fields associated with each resonant mode. The main goal of the experiments was a full experimental investigation and understanding of the E-field structures for all HOMs detected between $1.5 \mathrm{GHz}$ and $2.5 \mathrm{GHz}$ across both axes. The results of these studies are essential prior to finalising the cavity design and technical drawings for the construction of a superconducting radiofrequency (SCRF) cavity.

The series of tests was designed to confirm the validity of the modified experimental set-up as well as the results achieved in preliminary studies. ${ }^{14}$ This was also done to demonstrate the reproducibility of the data and verify the calibration of the equipment. During the tests, the dependence of the mode excitation on the coupler positioning (depth of insertion) was examined. This was necessary as VNA has finite power capabilities, and the efficient excitation of the modes is important to study low-Q modes. In Fig. 2, it can be seen that the modes' structures as measured are similar to those observed in Ref. 14, though with markedly improved magnitude of response in most cases. Additionally, we managed to remove the unphysical skew of the E-field towards one end of the axis for modes showing a weaker response. The skew of the E-field has been eliminated through fine-tuning of coupler position to ensure a uniform strength of coupling at either end of the cavity (this was not possible with the previous set-up). All the experiments presented here were carried out using the following procedure: every mode identified has been investigated with the bead running along one of the axes of the cavity with the couplers positioned either along the same or opposite axis. The axis along which the couplers have been located is referred to as an "active" axis, whilst the opposite axis is referred to as the "passive" axis. Once the measurements have been performed along one axis, the cavity would then 

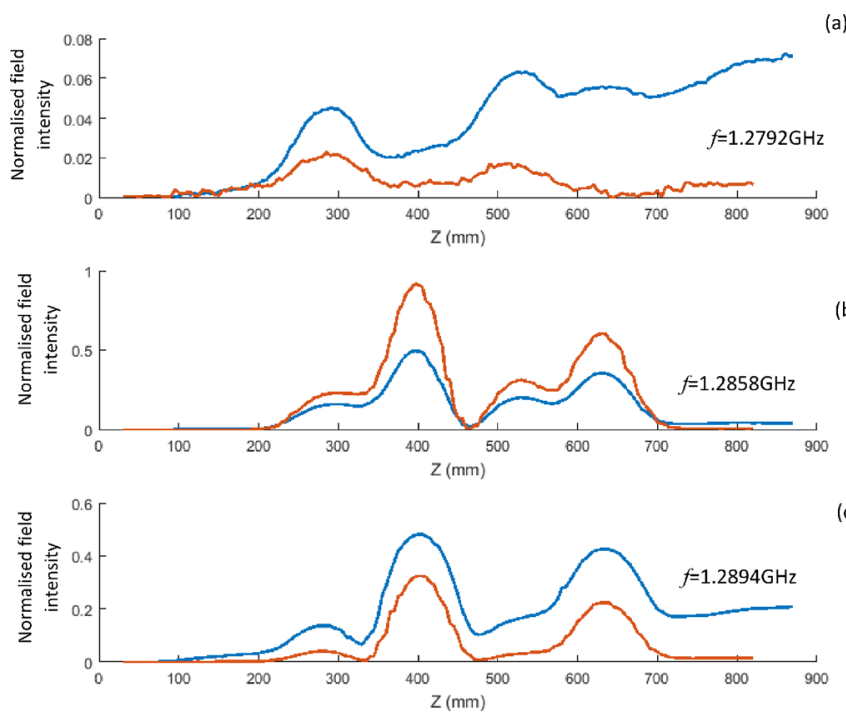

FIG. 2. Comparison of the pass-band eigenmodes' field structures as measured during the preliminary (blue lines) set of experiments ${ }^{14}$ and using the current set-up (red lines). The results are shown for modes at (a) $1.279 \mathrm{GHz}$, (b) $1.286 \mathrm{GHz}$, and (c) $1.289 \mathrm{GHz}$. The previous results ${ }^{14}$ display a bias towards one end while the set-up allows overcoming this issue; also, the noise to signal ratio has been improved.

be moved, ensuring that the position of the couplers did not change, so that the bead would run along the opposite (passive) axis, and the measurements repeated at the frequencies identified previously. First, the pass-band modes were identified and measured. Their structures are shown in Fig. 3 with the red solid line indicating the active axis, i.e., the axis along which the couplers were positioned while blue dotted line shows the field structure along the passive axis (no couplers, only the bead pull line). It was found that, while most of the modes have some degree of asymmetry, only the two pass-band modes observed at $1.274527 \mathrm{GHz}$ and 1.279228 $\mathrm{GHz}$ were shown to have a highly asymmetric structure
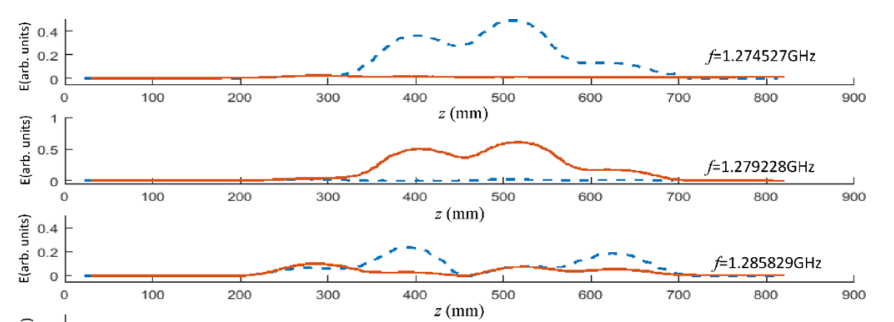

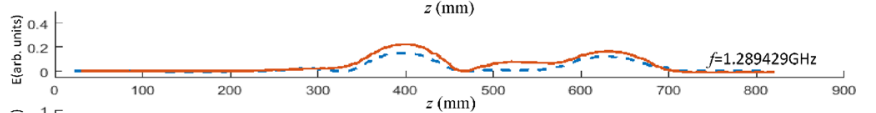

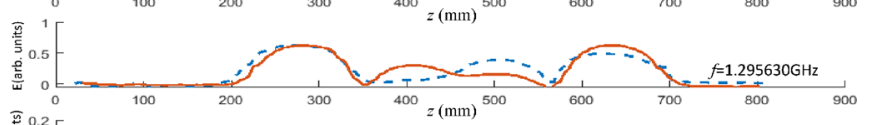
100

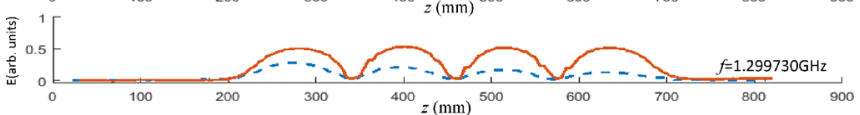

FIG. 3. A comparison of the pass-band mode structures on each axis of the cavity, with the couplers positioned on the axis 4-2 (red line), i.e., the axis 3-1 (Fig. 1) is passive. The figures show the modes having different degrees of asymmetricity with different fields on the different axes. The negative values of some of the residual fields outside the cavity come from the normalisation procedure, general asymmetry of the cavity, and losses over the cavity length.

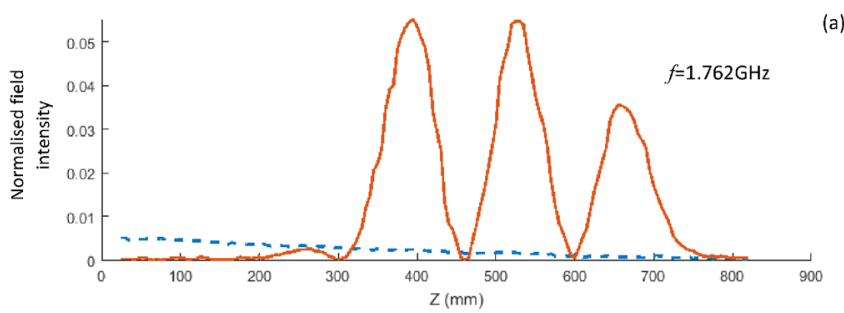

(a)

(b)

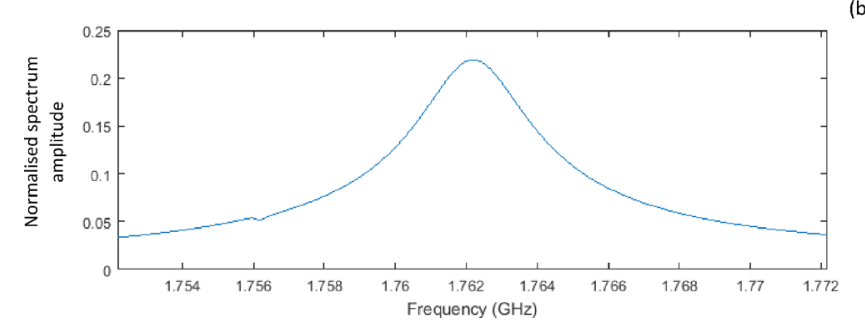

FIG. 4. (a) The results of the measurement of the HOM's (at $1.762 \mathrm{GHz}$ ) E field relative intensity along the centre of each axis [red line-active axis 4-2, blue is passive (3-1)]. (b) Zoom up frequency range $[1.752 \mathrm{GHz}$; $1.772 \mathrm{GHz}]$ of the normalised spectrum of the HOMs.

with the E-field almost entirely confined to a single axis of the cavity.

To carry out studies of HOMs, bead pull measurements and data analyses were carried out according to the same procedure described above. As before, the couplers and the bead were swapped between alternate axes. It was expected that all HOMs outside the pass band, i.e., above $1.3 \mathrm{GHz}$, would be localised to either one axis or the other, i.e., a high degree of asymmetry would be present. The experiments were carried out and typical results are shown in Figs. 4(a), $5(\mathrm{a})$, and 6 with blue and red lines showing results of measurements on the passive and active axes, respectively. Figures 4(b) and 5(b) illustrate the typical spectra of highorder eigenmodes as measured on the active [Fig. 4(b)] and passive [Fig. 5(b)] axes. We note some interesting features shown in Figs. 4(a) and 5(a). In Fig. 4(a), the expected mode structure is excited on the axis where the couplers are positioned, while in Fig. 5(a), the expected mode structure is excited on the passive axis, i.e., the couplers are aligned

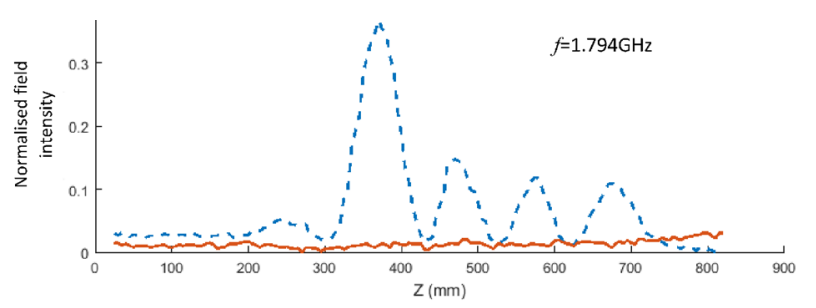

(a)

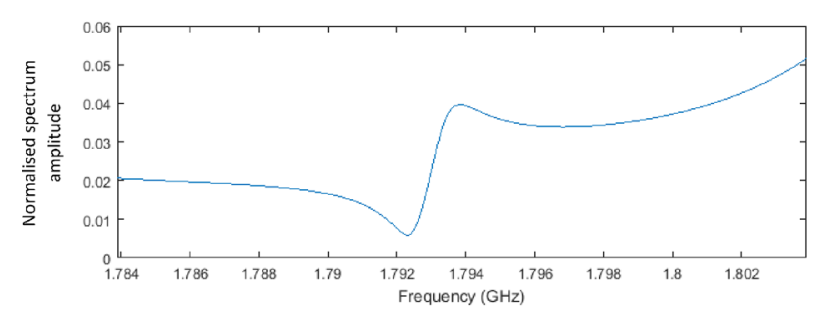

(b)

FIG. 5. (a) The results of the measurement of the HOM's (at $1.794 \mathrm{GHz}$ ) $\mathrm{E}$ field relative intensity along the centre of each axis [red line-active axis 4-2, blue is passive (3-1)]. (b) Zoom up frequency range $[1.784 \mathrm{GHz}$; $1.804 \mathrm{GHz}]$ of the normalised spectrum of the HOMs. 

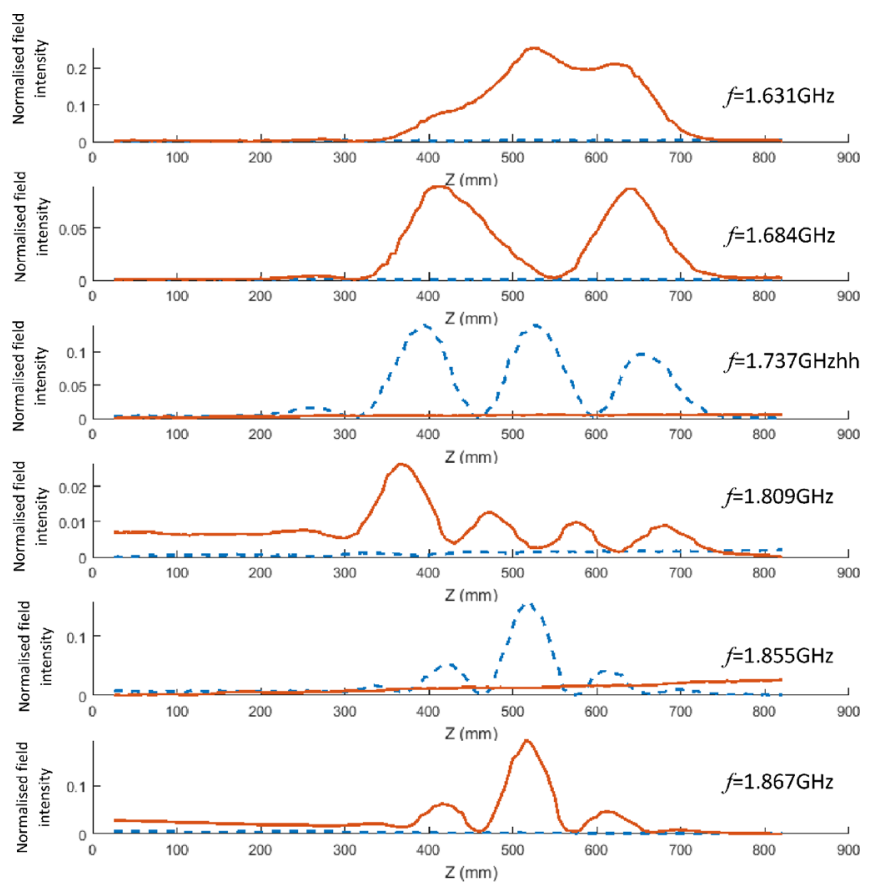

FIG. 6. The typical results of the measurements of the HOMs' electric field distribution in the frequency range [1.5 GHz to $2.5 \mathrm{GHz}]$. The E fields have been measured with couplers positioned on the axis 4-2 (red solid line) while the field measured on axis $3-1$ is indicated by the dashed blue line.

along the opposite axis with respect to the excited field localisation. This shows that asymmetry of the modes is irrelevant to the position of the couplers. While this shows that crossaxis excitation is possible in these experiments, it is not expected that electron bunches propagating along one of the axis will be able to excite the HOM confined to the other axis. This is due to zero coupling of the bunch to an asymmetric mode while propagating along the axis to which the mode is not confined, as the field amplitude on this axis will be zero. Similarly, for all other sets of experiments in which higher order modes above $1.3 \mathrm{GHz}$ were studied, we see that HOMs are not shared across both axes of the cavity, i.e., the field is almost entirely confined to one axis of the cavity or the other. The typical results for the field distributions of HOMs are illustrated in Fig. 6 where a set of five typical HOM field structures are shown in the frequency range from $1.5 \mathrm{GHz}$ to $2.5 \mathrm{GHz}$. As before, the dashed blue and solid red lines show the field distributions along the passive and active axes, respectively. From the results observed, we can see almost no evidence of higher order modes with similar responses across both axes, contrary to what was observed for some pass-band modes.

Overall, the results agree well with theoretical predictions and are as expected in terms of the mode structure and their distribution. We observed HOM excitation when excited by the couplers both on the same and opposite axis, but because the mode structure itself is largely confined to either the accelerating or decelerating set of cells in these cases, this is not a concern when implemented in an ERL. Indeed, as a zero field means no interaction of electromagnetic fields with an accelerating or decelerating electron, it is unlikely that such an asymmetric mode could be excited by a particle on the alternate axis. To conclude, we note that the dual-axis studies of the eigenmodes of the dual-axis prototype of the SCRF cavity have been carried out, and they show that, within the pass-band modes of the cavity, the excited operating mode has a highly symmetric structure and is not affected by the small changes to the cells' geometry. The remaining modes in the pass band have different degrees of asymmetry. This implies that energy recovery (and crossaxis transference) through excitation of the operating mode is feasible while the introduction of RF couplers to suppress other pass-band modes will be advantageous. The higher order modes have strongly asymmetric structures and are entirely confined to either one axis of the cavity or the other, minimising the possibility of cross-axis parasitic excitation. These results illustrate that this particular set-up could be a viable candidate for construction of a high average current ERL. The consistency of the preliminary theoretical studies from previous works with the results observed implies that the results obtained in these rigorous investigations are reliable and reputable. Further investigation could continue with RF studies of a cavity to improve the overall design of the cavity, e.g., reducing the field stress and R/Q of the modes in the pass-band, designing additional HOM couplers to further increase the BBU start current, as well as studying beam dynamics in such a system. Building upon the work presented, we conclude that the fundamental features predicted in the paper $^{13}$ have been confirmed and next steps such as optimisation of cavity parameters, construction of superconducting RF cavity, and RF testing of such a cavity are required to move forward with the development of a high average current ERL.

${ }^{1}$ D. W. Feldman, R. W. Warren, W. E. Stein, J. S. Fraser, G. Spalek, A. H. Lumpkin, J. M. Watson, B. F. Carlsten, H. Takeda, and T.-S. Wang, "Energy recovery in the Los Alamos free electron laser," Nucl. Instrum. Methods Phys. Res., A 259, 26 (1987).

${ }^{2}$ T. Nakamura, "Multi-turn circulation of an energy-recovery linac beam in a storage ring," Phys. Rev. Spec. Top. Accel. Beams 11, 032803 (2008).

${ }^{3}$ L. Merminga, D. R. Douglas, and G. A. Krafft, "High current energyrecovering electron LINACS,” Annu. Rev. Nucl. Part. Sci. 53, 387 (2003).

${ }^{4}$ G. H. Hoffstaetter and I. V. Bazarov, "Beam-breakup instability theory for energy recovery linacs,” Phys. Rev. Spec. Top. Accel. Beams 7, 054401 (2004).

${ }^{5}$ G. H. Hoffstaetter, I. V. Bazarov, and C. Song, "Recirculating beambreakup thresholds for polarized higher-order modes with optical coupling," Phys. Rev. Spec. Top. Accel. Beams 10, 044401 (2007).

${ }^{6}$ V. Ptitsyn, J. Beebe-Wang, I. Ben-Zvi, A. Fedotov, W. Fischer, Y. Hao, D. Kayran, V. N. Litvinenko, W. W. MacKay, C. Montag, E. Pozdeyev, T. Roser, D. Trbojevic, and N. Tsoupas, "ERHIC conceptual design," BNL Report No. BNL-90320-2009-CP.

${ }^{7} \mathrm{C}$. Xu and I. Ben-Zvi, "HOM frequency control of SRF cavity in high current ERLs,” Nucl. Instrum. Methods Phys. Res., A 883, 136-142 (2018).

${ }^{8}$ C.-X. Wang, "Conceptual design considerations of a 5-cell dual-axis SRF cavity for ERLs," in Proceedings of SRF2007 (Peking University, Beijing, China, 2007).

${ }^{9}$ S. U. De Silva, H. Park, J. R. Delayen, F. Marhauser, and A. Hutton, "Electromagnetic design of a superconducting twin axis cavity," in Proceedings of LINAC2016, MOPLR030, East Lansing, MI, USA, ISBN: 978-3-95450-169-4

${ }^{10}$ A. Seryi, "Ultrahigh flux compact x-ray source," patent PCT/GB2012/ 052632 (24 October 2012).

${ }^{11}$ R. Ainsworth, G. Burt, I. V. Konoplev, and A. Seryi, "Asymmetric superconducting rf structure," patent PCT/GB2015/053565 (24 November 2015).

${ }^{12}$ I. V. Konoplev, A. Seryi, A. J. Lancaster, K. Metodiev, G. Burt, and R. Ainsworth, "Compact, energy efficient superconducting asymmetric ERL for ultrahigh fluxes of X-ray and THz," AIP Conf. Proc. 1812, 100004 (2017).

${ }^{13}$ R. Ainsworth, G. Burt, I. V. Konoplev, and A. Seryi, “Asymmetric dual axis energy recovery linac for ultrahigh flux sources of coherent $\mathrm{X}$-ray and 
THz radiation: Investigations towards its ultimate performance," Phys. Rev. Accel. Beams 19, 083502 (2016).

${ }^{14}$ I. V. Konoplev, K. Metodiev, A. J. Lancaster, G. Burt, R. Ainsworth, and A. Seryi, Phys. Rev. Accel. Beams 20, 103501 (2017).

${ }^{15}$ R. Ainsworth, G. Boorman, A. Lyapin, S. Molloy, A. Garbayo, P. Savage, A. P. Letchford, and C. Gabor, "Bead-pull test bench for studying accelerating structures at RHUL," in Proceedings of the 2nd IPAC, San Sebastián, Spain (EPS-AG, Spain, 2011), p. MOPC049.

${ }^{16}$ S. H. Gold, A. Ting, V. Jabotinski, B. Zhou, and P. Sprangle, "Development of a high average current rf linac thermionic injector," Phys. Rev. Spec. Top. Accel. Beams 16, 083401 (2013).

${ }^{17}$ F. V. Hartemann, W. J. Brown, D. J. Gibson, S. G. Anderson, A. M. Tremaine, P. T. Springer, A. J. Wootton, E. P. Hartouni, and C. P. J. Barty, "High-energy scaling of Compton scattering light sources," Phys. Rev. Spec. Top. Accel. Beams 8, 100702 (2005).
${ }^{18}$ V. Cnudde and M. N. Boone, "High-resolution x-ray computed tomography in geosciences: A review of the current technology and applications," Earth-Sci. Rev. 123, 1 (2013).

${ }^{19}$ M. Tonouchi, "Cutting-edge terahertz technology," Nat. Photonics 1, 97 (2007).

${ }^{20}$ H.-T. Chen, W. J. Padilla, J. M. O. Zide, A. C. Gossard, A. J. Taylor, and R. D. Averitt, "Active terahertz metamaterial devices," Nature (London) 444, 597 (2006).

${ }^{21}$ G. L. Carr, M. C. Martin, W. R. McKinney, K. Jordan, G. R. Neil, and G. P. Williams, "High-power terahertz radiation from relativistic electrons," Nature (London) 420, 153 (2002).

${ }^{22}$ J. F. Federici, B. Schulkin, F. Huang, D. Gary, R. Barat, F. Oliveira, and D. Zimdars, "THz imaging and sensing for security applicationsexplosives, weapons and drugs," Semicond. Sci. Technol. 20, S266 (2005). 